\title{
Prevalência da lombalgia e sua repercussão anatomofuncional em adultos e idosos: Revisão sistemática
}

\author{
Prevalence of low back pain and its anatomical and fnctional approaches in adults and \\ elderly: A systematic review
}

Agnês Mie Sakamotoㄹ, Flávia Martins Gervásio², Daniela de Souza Silva³, Jéssyka Viana Valadares Franco ${ }^{4}$, Letícia Antunes Spindola 5

\section{RESUMO}

A lombalgia mecânica comum ou inespecífica é caracterizada pela dor intensa ou moderada na região entre as últimas costelas e nádegas. Buscou-se identificar na literatura os fatores condicionantes que favorecem o desencadeamento da lombalgia e sua relação com estruturas anatômicas e funcionalidade em adultos e idosos. Trata-se de uma revisão sistemática da literatura que permite a análise de estudos para aplicação na prática clínica visando compreender o tema a partir de estudos independentes. Nessa perspectiva, identificou que no sexo feminino há predominância na faixa etária superior a 40 anos, enquanto no sexo masculino a faixa etária de incidência é a partir dos 31 anos. As características recorrentes de dor foram movimentos laborais repetitivos, longos períodos na postura sentada, ausência de exercícios para ativação e relaxamento das estruturas musculoesqueléticas, sedentarismo. $O$ estudo demonstrou que os adultos divergem dos idosos no contexto etiológico das causas de lombalgia. Nos adultos os músculos são os principais afetado, seguido por estruturas articulares e ósseas, já nos idosos há predomínio dos processos de desgaste ósseos e articulares, sendo as mulheres, as mais acometidas. Uma análise crítica verifica que a condição socioeconômica determina a qualidade do tratamento e profilaxia, sendo medicamentos e fisioterapia as abordagens mais frequentes.

Palavras-chave: Lombalgia. Coluna lombar. Desempenho funcional.

\section{ABSTRACT}

Common or mechanical low back pain is characterized by severe or moderate pain in the region between the last ribs and buttock. We sought to identify in the literature the conditioning factors that favor the triggering of low back pain and its relationship with anatomical structures and functionality in adults and the elderly. It is a systematic review of the literature that allows the analysis of for application in clinical practice aiming to the theme from independent studies. In this perspective, he identified that in females there is a predominance in the age group above 40 years while in males, incidence age range is from 31 years. The recurrent characteristics of pain were repetitive labor movements, long periods of sitting, absence of exercises to activate and relax musculoskeletal structures, physical inactivity. The study showed that adults differ from the elderly in the etiological context of the causes of low back pain. In adults, muscles are the main affected followed by joint and bone structures, whereas in the elderly, bone and joint processes predominate, with women being the most affected. A critical analysis finds that socioeconomic status determines the quality of treatment, with drugs and physiotherapy being the most frequent approaches.

Keywords: low back pain. Lumbar spine. Physical Functional Performance.
Licenciada em Química pela Universidade Federal de Mato Grosso - UFMT, acadêmica de Medicina da Universidade de Gurupi - UNIRG.

E-mail:

miesakamoto@hotmail.com

Bacharel em Fisioterapia pela Universidade Estadual de Goiás, Docente na Universidade Estadual de Goiás (UEG), acadêmica de Medicina da Universidade de Gurupi-UNIRG.

E-mail:flavia.gervasio@ueg.br

${ }^{3}$ Bacharel em Enfermagem pelo Centro Universitário Tocantinense Presidente Antônio Carlos - UNITPAC. Enfermeira da Unidade de Pronto Atendimento de Palmas, acadêmica de Medicina da Universidade de Gurupi - UNIRG.

E-

mail:danielasouzaofs@gmail.com

Bacharel em $\begin{array}{r}\text { Farmácia } \\ \text { Centro }\end{array}$
Generalista pelo Tocantinense
Universitário
Presidente Antonio Carlos -
UNITPAC. Pós-graduanda lato
sensu em Farmácia Clínica e
Hospitalar, Citologia Oncótica e
Atenção Primária à Saúde com
Ênfase em Saúde da Família pela
Faculdade UniBF, acadêmica de
Medicina da Universidade de
Gurupi - UNIRG.
E-mail:
jessykavviana@gmail.com




\section{INTRODUÇĀO}

A lombalgia mecânica comum ou inespecífica, objeto deste estudo, é caracterizada pela dor intensa ou moderada na região compreendida entre as últimas costelas e as nádegas. Possui etiologia multifatorial tais como laborais, obesidade, síndromes depressivas, hábitos posturais, dentre outros. Estes fatores conduzem a alteração da estabilidade anatomofuncional com impacto na qualidade de vida e repercussões nas capacidades físico funcionais e sociais ${ }^{1,2,3}$.

A sobrecarga mecânica deve-se ao maior suporte do peso corporal recair sobre as vértebras lombares, cujo arcabouço anatômico é designado para suportar maior massa corpórea, bem como, constantes atividades dos músculos dorsais e abdominais para manter o tronco ereto. Adotando-se a postura sentada, esta carga é então distribuída para os membros inferiores por meio das tuberosidades isquiáticas, sendo esta postura a que apresentação maior índice de dor ${ }^{4}$. Assim o encurtamento, como dos músculos isquiotibiais e iliopsoas é considerado um fator desencadeante da sintomatologia dolorosa podendo acentuar a lordose lombar e ampliar a carga sobre a coluna e discos intervertebrais ${ }^{1,2}$.

A dor lombar tem se tornado um problema de saúde pública mundial e no Brasil cerca de 27 milhões de pessoas apresentam doenças da coluna vertebral ${ }^{1}$. A prevalência das lombalgias na população em geral é de $50 \%$ a $80 \%$, podendo atingir até $65 \%$ dos adultos e idosos anualmente. É possível afirmar em algum momento da vida até $84 \%$ das pessoas nestas faixas etárias apresentaram lombalgia ${ }^{5}$.

Com base em estudos científicos de observação é possível afirmar que o processo de envelhecimento atinge a coluna lombar por meio da desidratação e degeneração progressiva dos discos intervertebrais e das articulações zigoapofisárias, limitando assim, sua função de amenizar os impactos e manter a mobilidade e em decorrência disso a coluna começa a apresentar instabilidade progressiva e dor na região afetada, sendo esses os principais motivos pelos quais adultos e idosos são acometidos pela lombalgia ${ }^{4}$.

A dor lombar crônica ocorre em 76,7\% dos casos com impacto nas funções laborais, sendo um dos principais motivos de consultas médicas, emissão de atestados, laudos e hospitalizações ${ }^{5}$. Desta forma a patologia abordada é considerada a terceira causa de suspensão temporária no trabalho, bem como de aposentadorias prematuras, gerando alto custo para a sociedade e para os sistemas de saúde nos países desenvolvidos ${ }^{1,5,6,7}$. 
Em decorrência disso, gera-se uma expressiva necessidade de assistência que se situa entre as ações médicas, de reabilitação e de auto cuidado do paciente ${ }^{8}$.

Atualizar e ampliar os conhecimentos sobre a lombalgia permite que na prática clínica haja adequado diagnóstico e tratamento, adotando-se medidas precisas que permitam diferenciá-la de outros quadros clínicos confundidores, o que promove menor sobrecarga no sistema de saúde, com resolução do problema, além da recuperação do indivíduo, contribuindo para uma melhor qualidade de vida ${ }^{2}$.

Buscou-se identificar na literatura nacional os fatores condicionantes que favorecem o desencadeamento da lombalgia e sua relação com estruturas anatomofuncionais em adultos e/ou idosos.

\section{MATERIAIS E METODOS}

Trata-se de uma revisão sistemática da literatura, que permite a análise de estudos relevantes para aplicação na prática clínica, visando compreender determinado tema a partir de estudos independentes.

Esta revisão foi realizada em seis etapas: a identificação da pergunta norteadora, a busca ou amostragem da literatura, a coleta de dados, a análise crítica dos estudos incluídos, a discussão dos resultados e a apresentação da revisão.

A primeira etapa consistiu na elaboração da pergunta norteadora: Qual a prevalência de lombalgia entre adultos e idosos, com destaque aos principais fatores associados e estruturas anatômicas envolvidas?

A segunda etapa foi a busca da amostragem nas bases indexadas no portal de dados Biblioteca Virtual em Saúde (BVS) e United States National Library of Medicine (PubMED). Os descritores consultados no Decs da BVS e no Mesh da PubMed foram: adultos/adults; idoso/elderly; Coluna lombar/ Lumbar spine; Dor lombar/low back pain, estas características associadas ou não a tratamento conservador na modalidade fisioterapia. $O$ operador booleano utilizado foi AND. O quadro 1 representa as bases de dados e a combinação dos descritores.

Foram incluídos artigos que tiveram como foco principal a avaliação da coluna lombar associado a quadros de dor em adultos e/ou idosos, compreendendo o período de publicação de 2010 à 2020, no idioma português, cuja caracterização do desfecho estabeleça relação com aspectos anatômicos e/ou funcionais, abordagem terapêutica conservadora da coluna lombar, identificação de fatores associados a lombalgia. Os 
critérios de exclusão foram pesquisas que avaliaram coluna lombar associada a doenças crônico-degenerativas como artrose, espondilite anquilosante, artrite reumatoide, diagnóstico associado de hérnia discal ou lombociatalgia; pesquisas com crianças e/ou adolescentes até 18 anos, validação de escalas; análises de intervenções cirúrgicas; artigos duplicados e aqueles cujo o desenho metodológico apresentasse relato/série de casos, revisões de literatura, bem como os livros.

Os artigos triados nas bases de dados foram submetidos ao teste de relevância (quadro 1), que consiste na leitura dos títulos e resumos dos artigos pré-selecionados no intuito de estabelecer pré requisitos para a fase seguinte, que se deu com a leitura na íntegra dos artigos selecionados. A terceira e quarta etapa consistiram em extrair e analisar criticamente os estudos incluídos. Os principais resultados são apresentados no quadro 2 que expõe a síntese dos dados dos artigos. A quinta etapa consistiu na redação da discussão a despeito dos resultados das etapas anteriores. A sexta etapa é a apresentação da revisão integrativa.

Quadro 1: Base de dados e combinação dos descritores utilizados na busca dos artigos

\begin{tabular}{l|l}
\hline \multicolumn{1}{c|}{ Base de dados } & \multicolumn{1}{c}{ Descritores } \\
\hline \multirow{2}{*}{ BVS } & "Coluna lombar" AND "adulto"; "Coluna lombar" AND "idoso"; \\
\cline { 2 - 2 } & "lombalgia" AND "adulto"; "lombalgia" AND "idoso" \\
\hline \multirow{2}{*}{ PubMed } & "Lumbar spine" AND " adult", Lumbar spine" AND " elderly" \\
\cline { 2 - 2 } & "low back pain" AND "adult"; "low back pain" AND "elderly" \\
\hline
\end{tabular}

\section{RESULTADOS}

A pesquisa nas bases de dados identificaram 30 artigos, sendo que destes 18 foram excluídos. O principal fator de exclusão foi ser artigo de revisão, não identificar as estruturas anatomofuncionais relacionadas a lombalgia e serem estudos que descrevem métodos de avaliação da coluna vertebral.

Foram inclusos 12 artigos, onde predominou o estudo analítico transversal. Nessa perspectiva a revisão identificou que a faixa etária superior a 40 anos e o sexo feminino como prevalentes para lombalgia. Entre os homens a faixa etária de início dos sintomas é de 31 a 59 anos.

As características preditoras recorrentes de dor foram movimentos laborais repetitivos, longos períodos na postura sentada e sem pausas, ausência de exercícios para ativação e relaxamento das estruturas musculoesqueléticas e sedentarismo 2,4,9,10. 
Cabe ressaltar que as estruturas anatômicas mais acometidas pelas lombalgias são as osteomioarticulares. Nesta perspectiva, os músculos estabilizadores do tronco, tais como: multífido lombar, transverso do abdômen, longuíssimo torácico, fibras posteriores do oblíquo interno, quadrado lombar, fibras médias parte lombar do iliocostal são os primeiros a serem afetados, enquanto que as estruturas ósseas e articulares são as últimas a serem ${ }^{11}$.

Os resultados desta revisão são apresentados no quadro 2, contendo as informações de autor/ano, objetivos, classificação do tipo de estudo (desenho) e os principais desfechos.

Quadro 2: Estudos selecionados segundo autor/ano de publicação, objetivo/desenho do estudo, principais resultados e a anatomia relacionada.

\begin{tabular}{|c|c|c|c|}
\hline AUTOR/ANO & $\begin{array}{c}\text { OBJETIVOS/DESENH } \\
\text { O (D) }\end{array}$ & PRINCIPAIS RESULTADOS & $\begin{array}{c}\text { ANATOMIA } \\
\text { RELACIONADA }\end{array}$ \\
\hline $\begin{array}{l}\text { Rodrigues } \\
\text { I.S.A.; Oliveira } \\
\text { L.M.M.; } \\
\text { Fernandes } \\
\text { F.E.C.V.; et al } \\
\text { / } 201911\end{array}$ & $\begin{array}{l}\text { Avaliar a ocorrência de } \\
\text { lombalgia em pacientes } \\
\text { atendidos em uma } \\
\text { Unidade de Pronto } \\
\text { Atendimento e fatores } \\
\text { associados } \\
\text { D: Transversal }\end{array}$ & $\begin{array}{l}\text { Dentre os } 923 \text { pacientes com dor } \\
\text { aguda, } 261 \text { apresentaram dor lombar, } \\
\text { com idade média de } 39,7 \text { anos. Os } \\
\text { homens representam mais da metade } \\
\text { dos atendimentos. A busca pelo } \\
\text { atendimento na UPA é espontâneo e } \\
\text { normalmente sem encaminhamento }\end{array}$ & $\begin{array}{l}\text { Musculos } \\
\text { eretores da } \\
\text { espinha, discos } \\
\text { intervertebrais, } \\
\text { articulação } \\
\text { sinovial plana da } \\
\text { lombar }\end{array}$ \\
\hline $\begin{array}{l}\text { Ferreira M.S.; } \\
\text { Navega M.T. / } \\
2010^{2}\end{array}$ & $\begin{array}{l}\text { Implantar um programa } \\
\text { de "Escola da Postura" } \\
\text { para pacientes com } \\
\text { lombalgia crônica. } \\
\text { D: Experimental }\end{array}$ & $\begin{array}{l}\text { O programa resultou melhora } \\
\text { significativa na capacidade funcional, } \\
\text { dor, estado geral de saúde, vitalidade e } \\
\text { aspectos sociais, com predomínio em } \\
\text { mulheres entre } 12 \text { a } 46 \text { anos, sem } \\
\text { apresentar mudanças no aspecto } \\
\text { emocional e físico. }\end{array}$ & $\begin{array}{l}\text { Articulação } \\
\text { sinovial plana da } \\
\text { região lombar }\end{array}$ \\
\hline $\begin{array}{l}\text { Barros S.S.; } \\
\text { Ângelo Urâa } \\
\text { R.C.O.; Uchôa } \\
\text { E.P.B.L / } \\
2011^{4}\end{array}$ & $\begin{array}{l}\text { Investigar a provável } \\
\text { relação entre lombalgia } \\
\text { e atividades laborais na } \\
\text { posição sentada. } \\
\text { D: Transversal }\end{array}$ & $\begin{array}{l}\text { A dor lombar realçou em mulheres com } \\
\text { faixa etária superior a } 40 \text { anos, maior } \\
\text { tempo de trabalho na instituição e } \\
\text { sedentarismo. Enquanto IMC e } \\
\text { intervalos durante a jornada não se } \\
\text { apresentam como elementos de riscos. }\end{array}$ & $\begin{array}{l}\text { Articulação } \\
\text { sinovial plana da } \\
\text { região lombar }\end{array}$ \\
\hline $\begin{array}{l}\text { Freitas K.P.N.; } \\
\text { Barros S.S.; } \\
\text { Ângelo } \\
\text { R.C.O.; Uchôa } \\
\text { E.P.B.L / } \\
2011^{9}\end{array}$ & $\begin{array}{l}\text { Verificar os efeitos da } \\
\text { cinesioterapia em } \\
\text { pacientes portadores de } \\
\text { lombalgia ocupacional. } \\
\text { D: Transversal/ } \\
\text { analítico }\end{array}$ & $\begin{array}{l}\text { A lombalgia da postura laboral sentada } \\
\text { predominou no gênero feminino, faixa } \\
\text { etária superior a } 41 \text { anos. Houve } \\
\text { expressiva redução da dor após a } \\
\text { fisioterapia clássica, com melhora na } \\
\text { amplitude de movimento e capacidade } \\
\text { funcional. Havendo redução da } \\
\text { incidência de lombalgia após a adoção } \\
\text { do programa de exercícios. }\end{array}$ & $\begin{array}{l}\text { Coluna vertebral, } \\
\text { músculos } \\
\text { estabilizadores } \\
\text { do tronco, } \\
\text { multífido lombar, } \\
\text { transverso do } \\
\text { abdômen, } \\
\text { longuíssimo } \\
\text { torácico, fibras } \\
\text { posteriores do } \\
\text { oblíquo interno. }\end{array}$ \\
\hline $\begin{array}{l}\text { Romero D.E.; } \\
\text { Muzy J.; Maia } \\
\text { L.; Marques } \\
\text { A.P.; Souza } \\
\text { Júnior P.R.B.; }\end{array}$ & $\begin{array}{lr}\text { Descrever os } & \text { principais } \\
\text { tratamentos } & \text { para } \\
\text { lombalgia crônica, } \\
\text { análise dos fatores } \\
\text { associados e a } \\
\text { distinção na utilização }\end{array}$ & $\begin{array}{l}\text { Maior incidência de lombalgia crônica } \\
\text { ocorre em mulheres entre } 18 \text { a } 60 \text { anos, } \\
\text { com baixa condição socioeconômica, } \\
\text { sem plano de saúde e condições de } \\
\text { saúde desfavoráveis. A intervenção } \\
\text { mais usual é a medicamentosa, e em }\end{array}$ & $\begin{array}{l}\text { Coluna vertebral, } \\
\text { lesão parcial dos } \\
\text { discos } \\
\text { intervertebral }\end{array}$ \\
\hline
\end{tabular}


DOI: 10.18606/2318-1419/amazonia.sci.health.v8n3p106-117 SAKAMOTO, AM. GERVÁSIO, FM, SILVA, DS. FRANCO, JVV. Revista Amazônia Science \& Health SPINDOLA, LA.

ISSN: 2318-1419

2020, Vol. 8, №3

Prevalência da lombalgia e sua repercussão anatomofuncional em adultos e idosos: Revisão sistemática

\begin{tabular}{|c|c|c|}
\hline $\begin{array}{l}\text { Castanheira } \\
\text { D. / } 2019^{10}\end{array}$ & $\begin{array}{l}\text { de fisioterapia ou } \\
\text { remédios } \\
\text { D: Transversal }\end{array}$ & $\begin{array}{l}\text { segundo plano a abordagem } \\
\text { fisioterapêutica. }\end{array}$ \\
\hline $\begin{array}{l}\text { Barbosa } \\
\text { F.S.S.; } \\
\text { Almeida } \\
\text { C.C.R.; } \\
\text { Gonçalves M. } \\
\text { Análise } \\
2010^{12}\end{array}$ & $\begin{array}{l}\text { Identificar e analisar o } \\
\text { comportamento da } \\
\text { fadiga do músculo } \\
\text { eretor da espinha em } \\
\text { um teste de resistência } \\
\text { isométrica até a } \\
\text { exaustão. } \\
\text { D: Experimental }\end{array}$ & $\begin{array}{l}\text { Homens universitários na faixa etária } \\
\text { de } 20 \text { anos apresentam resistência } \\
\text { isométrica do eretor da espinha } \\
\text { inversamente proporcional a } \\
\text { intensidade de contração, a qual não } \\
\text { promove efeito sobre a taxa de declínio } \\
\text { da fadiga muscular do músculo eretor } \\
\text { da espinha direito e esquerdo. }\end{array}$ \\
\hline $\begin{array}{l}\text { Pereira Silva } \\
\text { J, Jesus- } \\
\text { Moraleida F, } \\
\text { Felício DC, } \\
\text { Queiroz BZ, } \\
\text { Ferreira ML, } \\
\text { Pereira LSM / } \\
2019^{13}\end{array}$ & $\begin{array}{l}\text { Avaliar a capacidade do } \\
\text { exame de força de } \\
\text { pressão manual prever } \\
\text { dor lombar aguda em } \\
\text { mulheres idosas } \\
\text { D: Transversal }\end{array}$ & $\begin{array}{l}\text { Fatores associados a uma } \\
\text { incapacidade físico funcional são: maior } \\
\text { intensidade de dor, rigidez matinal na } \\
\text { coluna lombar, dificuldade para dormir, } \\
\text { pior mobilidade funcional e saúde física, } \\
\text { maiores níveis de índice de massa } \\
\text { corporal, cinesiofobia e pior saúde } \\
\text { mental }\end{array}$ \\
\hline
\end{tabular}

Verificar a eficácia da Garcia A.N.; fisioterapia na dor, ação Gondo F.L.B.; funcional e amplitude Costa R.A.; et do movimento de flexão al $/ 2011^{14}$ de coluna

D: Ensaio clínico

Segundo

S.T.S.P;

Valesin Filho

E.S.; Lenza

M.; et al / $2016^{13}$

\begin{tabular}{|c|c|}
\hline . & $\begin{array}{l}\text { coluna lombar. } \\
\text { D: Transversal }\end{array}$ \\
\hline $\begin{array}{l}\text { Ferreira } \\
\text { M.S.M.; } \\
\text { Pereira M.G. / } \\
2016^{2}\end{array}$ & $\begin{array}{l}\text { Identificar o efeito } \\
\text { moderador do "Tipo de } \\
\text { Família" na relação } \\
\text { entre a incapacidade } \\
\text { funcional e a qualidade } \\
\text { de vida em doentes } \\
\text { com lombalgia crônica. } \\
\text { D: Quantitativo e } \\
\text { transversal }\end{array}$ \\
\hline
\end{tabular}

Eichinger

F.L.F.; Soares

A.V.; Carvalho

Júnior J.M.; et

al / $2016^{17}$
Mensurar

reprodutibilidade

interobservador da

avaliação radiográfica

da instabilidade da coluna lombar.

D: Transversal

\section{Propor}

valores

normativos para o teste

lombar.

D: Transversal de dinamometria
Há melhora dos níveis de dor, desempenho funcional e amplitude de movimento de flexão de coluna lombar em adultos de ambos os gêneros com idade entre 18 e 80 anos, com dor lombar crônica submetidos aos métodos McKenzie e Back School.

Para o nível L4/L5, adotou-se como instáveis os valores dinâmicos de translação vertebral divergentes. Resultando em casos de instabilidade sagital em mulheres entre 30 a 50 anos, como também casos de instabilidade angular ou rotacional, assumindo diferenças angulares entre extensão e flexão para L4/L5 pacientes com lombalgia crônica, com média de idade de 51 a 64 anos, predomínio do sexo feminino. Evidenciou que a relação entre incapacidade funcional e qualidade de vida é mais acentuado para as famílias com dificuldades no que diz respeito a autonomia e identidade do que nas mais próximas emocionalmente.

A força muscular média do sexo masculino (média 27,1 anos) é de114,0 $\operatorname{kgf}( \pm 25,4)$, sendo superior ao feminino (média 28,2 anos) registado em 48,5 $\mathrm{kgf}$. A valência força relacionou-se tanto à saúde quanto ao desempenho físico em diferentes faixas etárias.
Participaram da pesquisa 213

\section{Músculos}

lombares no nível L4 e L5, articulação sinovial plana da região lombar.

Componentes musculoesqueléti cos da coluna lombar-

articulações a curvatura vertebral.

Componentes musculoesqueléti cos da coluna lombar articulações

músculos anterolaterais e posteriores de tronco.

Articulações sinovial plana da coluna lombar (L4 e L5).

Coluna lombar, inflamação dos músculos eretores espinha. da

Coluna lombar, músculos eretores da espinha 


\begin{tabular}{|c|c|c|c|}
\hline $\begin{array}{lr}\text { Silva } & \text { TPD, } \\
\text { Araújo } & \text { WN, } \\
\text { Stival } & \text { MM, } \\
\text { Toledo } & \text { AM, } \\
\text { Burke } & \text { TN, } \\
\text { Carregaro } & \text { RL / } \\
2018^{18} & \end{array}$ & $\begin{array}{l}\text { Avaliar o desconforto } \\
\text { muscoloesquelético, a } \\
\text { capacidade laboral e a } \\
\text { fadiga residual de } \\
\text { enfermeiros que } \\
\text { trabalham em ambiente } \\
\text { hospitalar. } \\
\text { D: Transversal }\end{array}$ & $\begin{array}{l}\text { Houve um predomínio de mulheres } \\
\text { entre } 31 \text { a } 40 \text { anos, casadas, com filhos } \\
\text { e com carga horária no hospital de } \\
\text { 40h/semanais. Além disso, houve } \\
\text { supremacia de dor na região lombar e } \\
\text { torácica e presença de fadiga. Contudo, } \\
\text { a associação entre fadiga e a presença } \\
\text { de desconforto não foi significante. }\end{array}$ & $\begin{array}{l}\text { Músculos } \\
\text { lombares desvios } \\
\text { das curvaturas } \\
\text { vertebrais. }\end{array}$ \\
\hline
\end{tabular}

\section{DISCUSSÃO}

Identificou-se que a incidência de lombalgia acomete predominantemente o sexo feminino com faixa etária superior a 40 anos $^{2,4,9,10}$, e o sexo masculino, de menor prevalência, predomina entre 31 a 59 anos ${ }^{1}$. Os dados epidemiológicos em relação à idade corroboram com os achados dessa revisão cujo predomínio entre homens localizam-se acima de 40 anos e nas mulheres entre 50 a 60 anos ${ }^{11-19}$, ou seja, em termos etários a queixa inicia-se precocemente em homens.

A lombalgia acomete de 70 a $80 \%$ da população adulta em momentos produtivos da vida 6-7, sendo a segunda dor mais frequente nos indivíduos ${ }^{20}$. É a sétima condição mais comum em pacientes que buscam as Unidades Básicas de Saúde (UBS), além de ser considerada uma condição musculoesquelética periódica ${ }^{21}$.

O predomínio de lombalgia ocorre mais no adulto do que no idoso. Neste último, a lombalgia associa-se a tumores, traumas vertebrais por osteoporose, infecções, estenose do canal medular e lombalgia mecânica ${ }^{22}$. Por consequência, a lombalgia em idosos causa dificuldade para dormir, rigidez na coluna lombar principalmente matinal, mobilidade funcional diminuída, pior saúde física, cinesiofobia, auto índice em quedas ${ }^{13}$.

A revisão identificou como causas prevalentes para a lombalgia o desgaste e/ou trauma na coluna vertebral, a adoção de sobrecarga mecânica e esforços físicos, a postura incorreta e o sedentarismo ${ }^{1,4,9}$.

A sintomatologia da lombalgia é dor associada a diminuição da força muscular, restrição da amplitude de movimento, espasmos musculares e alterações posturais ${ }^{11}$.

A dor lombar pode ser classificada conforme sua duração em aguda com persistência de menos de 4 semanas, subaguda com menos de 12 semanas e crônica acima de 12 semanas ${ }^{23}$.

A dor lombar crônica apresenta comprometimento no cumprimento das funções laborais, com incidência em 76,7\% dos trabalhadores ${ }^{11}$. Iguti e Hoehne ${ }^{24}$ averiguaram 6500 
trabalhadores da construção civil, sendo que 1262 apresentaram lombalgias, cujas principais causas foram esforços físicos (31\%), esforços em flexão (25\%), quedas (18\%), traumas diretos $(15 \%)$, sobrecargas $(5,3 \%)$.

A classificação da lombalgia mecânica segundo Helfenstein Junior, Goldenfum e Siena ${ }^{11}$ caracteriza-se de acordo com o comprometimento de origem muscular e ligamentar manifesto por fadiga muscular paravertebral e distensão; quanto a mobilidade e estabilidade da coluna exemplificado pela capacidade de torção da coluna lombar, bem como pelo ritmo lombo-pélvico que pode gerar instabilidade articular. Estes contextos porem associar-se e convergir com quadros psicossomáticos.

Especificamente em relação as estruturas anatômicas mais acometidas destacam-se a região de transição entre a coluna torácica e lombar e os espaços interespinhosos, a transição entre as vértebras L3, L4 e L5 e os respectivos discos intervertebrais ${ }_{2}$ músculos eretores da espinha, músculo profundos-estabilizadores do tronco (transverso do abdômen, multífidos lombar e oblíquos internos), corroborando com Macedo 20.

A correlação entre a força muscular e a incidência de lombalgia é inversamente proporcional, quanto maior a resistência da musculatura lombar menor será à frequência da dor lombar ${ }^{17}$.

O tratamento para lombalgias mecânicas visa aliviar a dor, evitar a recidiva, cada vez mais frequente e dolorosa, e às alterações anatômicas que evoluem com agravo. Os objetivos terapêuticos de cada fase da dor lombar devem considerar a capacidade socioambiental e financeira do paciente, o grau de comprometimento da coluna bem como, 0 acesso às formas terapêuticas ${ }^{25}$.

Afirma-se que as medidas de tratamento e profilaxia auxiliam na redução da dor e na perspectiva de evitar a reincidência de novos quadros, especialmente com a adoção de reabilitação e reeducação postural. Destacando-se os alongamentos, a natação, a acupuntura, o método pilates, as caminhadas, a massagem, a crioterapia, o calor e as atividades aeróbias 16,24,26,27,28.

Perante as condições socioeconômicas, a população mais acometida com a lombalgia crônica são do sexo feminino, condições socioeconômicas vulneráveis e/ou baixas, sem plano de saúde e circunstâncias desfavoráveis para uma boa qualidade de vida no âmbito da saúde ${ }^{10}$.

Comparando as situações socioeconômicas, diferentes tratamentos são designados. Medicamento são prioritários para grupos econômicos baixos, e fisioterapia e acupuntura 
para grupos econômicos de médio à alto. A abordagem terapêutica impacta diretamente na qualidade de vida, uma vez que os acometimentos costumam reincidir e associar-se aos aspectos biopsicossociais ${ }^{10,29}$.

A duração e a incapacidade que as lombalgias causam são extremamente variáveis entre as pessoas. Assim, não há um modelo de comportamento comum. Além disso, devese sobressair que, em variados graus, as estruturas anatômicas podem gerar dor por tempos diversos, sendo incapacitante ou não. Em vista disso, é normal suceder limitações funcionais em práticas cotidiana, em atividades relacionadas à profissão e lazer, restringindo à convivência do indivíduo.

A lombalgia apresenta impacto sobre a cadeia produtiva pois pode levar trabalhadores a licenças médicas, internações, incapacidade parcial ou total - aposentadoria precoce, implicando diretamente na economia, principalmente nos cofres públicos. Assim sendo, a relevância social apresentada por este estudo é substancial para nortear ações de saúde do trabalhador e ações epidemiológicas na abordagem preventiva para a lombalgia ${ }^{18}$.

Por fim a literatura estudada, qual sejam os artigos selecionados, apontam a existência de dor lombar em crianças e adolescentes, bem como grávidas e em pessoas com doenças neurodegenerativas, porém esta revisão buscou a condição do adulto e do idoso, as estruturas anatomofuncionais interligadas e a caracterização da lombalgia e tratamento e seu reflexo na vida produtiva e social dos indivíduos para efetivar o presente estudo.

\section{CONSIDERAÇOES FINAIS}

O presente estudo evidenciou que quanto aos adultos no que se refere ao contexto etiológico das causas de lombalgia, estes divergem dos idosos, sendo as mulheres as mais acometidas. Os músculos são o principal componente afetado nos quadros de lombalgia, seguido por estruturas articulares e ósseas respectivamente. Em relação aos fatores etiológicos mais frequentes os esforços repetitivos como um dos principais fatores mecânicos. O impacto gerado pelas dores lombares com limitação do movimento para execução das atividades diárias e laborais tem reflexo negativo no setor da economia. Uma análise crítica verifica que a condição socioeconômica determina a qualidade do tratamento e a profilaxia, sendo medicamentos e fisioterapia as abordagens mais frequentes. 


\section{REFERÊNCIAS}

1 Rodrigues ISA, Oliveira LMM, Fernandes FECV; Teles, MEV, Sena VS. Ocorrência de Lombalgia em una Unidade de Pronto Atendimento. Rev. Pesqui. 2019; 11(3): 823-7. Available from: http://ciberindex.com/c/ps/P113823

2 Ferreira MS, Navega MT. Efeitos de um programa de orientação para adultos com lombalgia. Acta ortop. bras. 2010; 18(3): 127-131. Available from:

http://www.scielo.br/scielo.php?script=sci_arttext\&pid=S1413$78522010000300002 \&$ Ing=en.

3 Brazil AV, Ximenes AC, Radu AS, Fernades AR, Appel C, Maçaneiro CH et al. Diagnóstico e tratamento das lombalgias e lombociatalgias. Rev. Bras. Reumatol. 2004; 44(6): 419-425. Available from:

http://www.scielo.br/scielo.php?script=sci_arttext\&pid=S0482$50042004000600005 \&$ Ing=en.

4 Barros SS, Ângelo RCO, Uchôa ÉPBL. Lombalgia ocupacional e a postura sentada. Rev. dor. 2011; 12(3): 226-230. Available from: http://www.scielo.br/scielo.php?script=sci_arttext\&pid=S1806$00132011000300006 \&$ Ing=en.

5 Nascimento PRC, Costa LOP. Prevalência da dor lombar no Brasil: uma revisão sistemática. Cad. Saúde Pública. 2015; 31( 6 ): 1141-1156. Available from: http://www.scielo.br/scielo.php?script=sci_arttext\&pid=S0102311 X2015000601141\&lng=en.

6 Andrade SC, Araújo AGR, Vilar MJP. Escola de Coluna: revisão histórica e sua aplicação na lombalgia crônica. Rev. Bras. Reumatol. 2005; 45(4): 224-228. Available from: http://www.scielo.br/scielo.php?script=sci_arttext\&pid=S048250042005000400006\&lng=en.

7 Leite A, Santos L, Araújo M, Cavalcante Neto J. Dor lombar e exercício físico: uma revisão sistemática. Rev Baiana de Saúde Pública. 2015; 39. 442-459. Available from: doi:10.5327/Z0100-0233-2015390200019.

8 Alrwaily M, Timko M, Schneider M, Stevans J, Bise C, Hariharan K, Delitto A. Treatmentbased classification system for low back pain: revision and update. Physical therapy. 2016; 96(7), 1057-1066. Available from: https://academic.oup.com/ptj/article/96/7/1057/2864925

9 Freitas KPN, Barros SS, Ângelo RCO, Uchôa ÉPBL. Lombalgia ocupacional e a postura sentada: efeitos da cinesioterapia laboral. Rev. dor. 2011; 12(4): 308-313. Available from: http://www.scielo.br/scielo.php?script=sci_arttext\&pid=S1806$00132011000400005 \&$ Ing=en.

10 Romero DE Muzy J, Maia L, Marques AP, Souza Júnior PRB, Castanheira D. Desigualdades e fatores associados ao tratamento do problema crônico de coluna no Brasil. Ciênc. saúde coletiva. 2019; 24( 11 ): 4211-4226. Available from: http://www.scielo.br/scielo.php?script=sci_arttext\&pid=S1413$81232019001104211 \&$ Ing=en.

11 Helfenstein Junior M, Goldenfum MA, Siena C. Lombalgia ocupacional. Rev. Assoc. Med. Bras. 2010; 56( 5 ): 583-589. 
12 Barbosa FSS, Almeida CCR, Gonçalves Mo. Análise espectral do sinal

eletromiográfico do músculo eretor da espinha obtido do teste de Sorensen. Fisioter. mov. (Impr.) 2010; 23(4 ): 575-583. Available from:

http://www.scielo.br/scielo.php?script=sci_arttext\&pid=S0103-

$51502010000400008 \&$ lng=en.

13 Pereira Silva J, Jesus-Moraleida F, Felício DC, Queiroz BZ, Ferreira ML, Pereira LSM. Fatores biopsicossociais associados com a incapacidade em idosos com dor lombar aguda: estudo BACE-Brasil. Ciênc. saúde coletiva. 2019; 24( 7 ): 2679-2690. Available from: http://www.scielo.br/scielo.php?script=sci_arttext\&pid=S141381232019000702679\&Ing=en. Epub July 22, 2019.

14 Garcia AN, Gondo FLB, Costa RA, Cyrillo FN, Costa LOP. Efeitos de duas intervenções fisioterapêuticas em pacientes com dor lombar crônica não-específica: viabilidade de um estudo controlado aleatorizado. Rev. bras. fisioter. 2011; 15(5): 420427. Available from: http://www.scielo.br/scielo.php?script=sci_arttext\&pid=S141335552011000500013\&lng=en.

15 Segundo STSP, Valesin Filho ES, Lenza M, Santos DCB, Rosemberg LA, Ferretti M. Reprodutibilidade interobservadores da avaliação radiográfica de instabilidade em coluna lombar. Einstein (São Paulo) . 2016; 14( 3 ): 378-383. Available from:

http://www.scielo.br/scielo.php?script=sci_arttext\&pid=S167945082016000300378\&lng=en.

16 Ferreira MSM, Pereira MG. O papel moderador do tipo de família na relação entre incapacidade funcional e qualidade de vida em doentes com lombalgia crônica. Ciênc. saúde coletiva. 2016; 21( 1 ): 303-309. Available from:

http://www.scielo.br/scielo.php?script=sci_arttext\&pid=S1413$81232016000100303 \& \operatorname{lng}=$ en.

17 Eichinger FLF, Soares AV, Carvalho Júnior JM, Gevaerd MS, Domenech, SC, Borges Junior, NG. Dinamometria lombar: um teste funcional para o tronco. Rev. Bras. Med. Trab. 2016; 14, 120-126. Available from:

http://www.anamt.org.br/site/upload_arquivos/revista_brasileira_de_medicina_do_trabalho _volume_14_n\%C2\%BA_2_31820169057055475.pdf

18 Silva TPD, Araújo WN, Stival MM, Toledo AM, Burke TN, Carregaro RL. Desconforto musculoesquelético, capacidade de trabalho e fadiga em profissionais da enfermagem que atuam em ambiente hospitalar. Rev. esc. enferm. USP. 2018; 52: e03332. Available from: http://www.scielo.br/scielo.php?script=sci_arttext\&pid=S008062342018000100420\&lng=en.

19 Leite DAT. Lombalgia e avaliação da incapacidade. Curitiba. Tese de Monografia Especialização em Medicina do Trabalho - Universidade Federal do Paraná 2016

20 Macedo DDP. Lombalgias. Cienc. Cult. [Internet]. 2011; 63(2 ): 42-44. Available from: http://cienciaecultura.bvs.br/scielo.php?script=sci_arttext\&pid=S0009-

$67252011000200013 \&$ Ing=en.

21 Branco PM, Dias CM, Fernandes L, Babulal J, Marques TP, Martinho G, et al. Lombalgia: Uma Condição Comum em Cuidados de Saúde Primários - Trabalho de Revisão [8 Congresso Nacional: idoso. 2014; Lisboa, Portugual 
22 Alves LS, Köbe BM, Frota B, Ziegler M. Lombalgia no idoso: emergência?. Acta méd.Porto Alegre. 2014, 7-7.

23 Imamura S, Kaziyama H, Imamura M. Lombalgia. Rev. Med. (São Paulo).2001;80:37590. Available from: http://www.periodicos.usp.br/revistadc/article/view/70000

24 Iguti AM, Hoehne EL. Lombalgias e trabalho. Rev. bras. saúde ocup. 2003; 28( 107108 ): 73-89. Available from:

http://www.scielo.br/scielo.php?script=sci_arttext\&pid=S0303-

76572003000200007\&lng=en.

25 Miateli Pires RA, Dumas FVL. Lombalgia: revisão de conceitos e métodos de tratamentos. Universitas: Ciências da Saúde. Centro de Ensino Unificado de Brasilia; 2009 Jul 23;6(2). Available from: http://dx.doi.org/10.5102/ucs.v6i2.718

26 Tobo A, Khouri ME, Cordeiro Q, Lima MC, Junior CAB, Battistella LR. Estudo do tratamento da lombalgia crônica por meio da Escola de Postura. Acta Fisiátr. 2010;17(3):112-116

27 Conceição JS, Mergener CR. Eficácia do método Pilates no solo em pacientes com lombalgia crônica: relato de casos. Rev. dor. 2012; 13(4): 385-388. Available from: http://www.scielo.br/scielo.php?script=sci_arttext\&pid=S1806$00132012000400015 \& \operatorname{lng}=$ en.

28 França FJR, Burke TN, Claret DC, Marques AP. Estabilização segmentar da coluna lombar nas lombalgias: uma revisão bibliográfica e um programa de exercícios. Fisioter. Pesqui. 2008; 15( 2 ): 200-206. Available from:

http://www.scielo.br/scielo.php?script=sci_arttext\&pid=S180929502008000200015\&lng=en.

29 Cozzensa da Silva M. Lombalgia em adultos de pelotas: prevalência e fatores de risco. Pelotas. Tese de Mestrado - Programa de Pós-graduação em Epidemiologia. Universidade de Pelotas. Dezembro de 2002. 